Supplementary Online Material

\title{
Axial heterostructures with phase-controlled metastable segments via post-growth reactions of $\mathrm{Ge}$ nanowires
}

Eli Sutter ${ }^{1}$ and Peter Sutter ${ }^{2}$

${ }^{1}$ Department of Mechanical and Materials Engineering, University of Nebraska-Lincoln, Lincoln, NE 68588 (USA)

${ }^{2}$ Department of Electrical and Computer Engineering, University of Nebraska-Lincoln, Lincoln, $N E$ 68588 (USA)

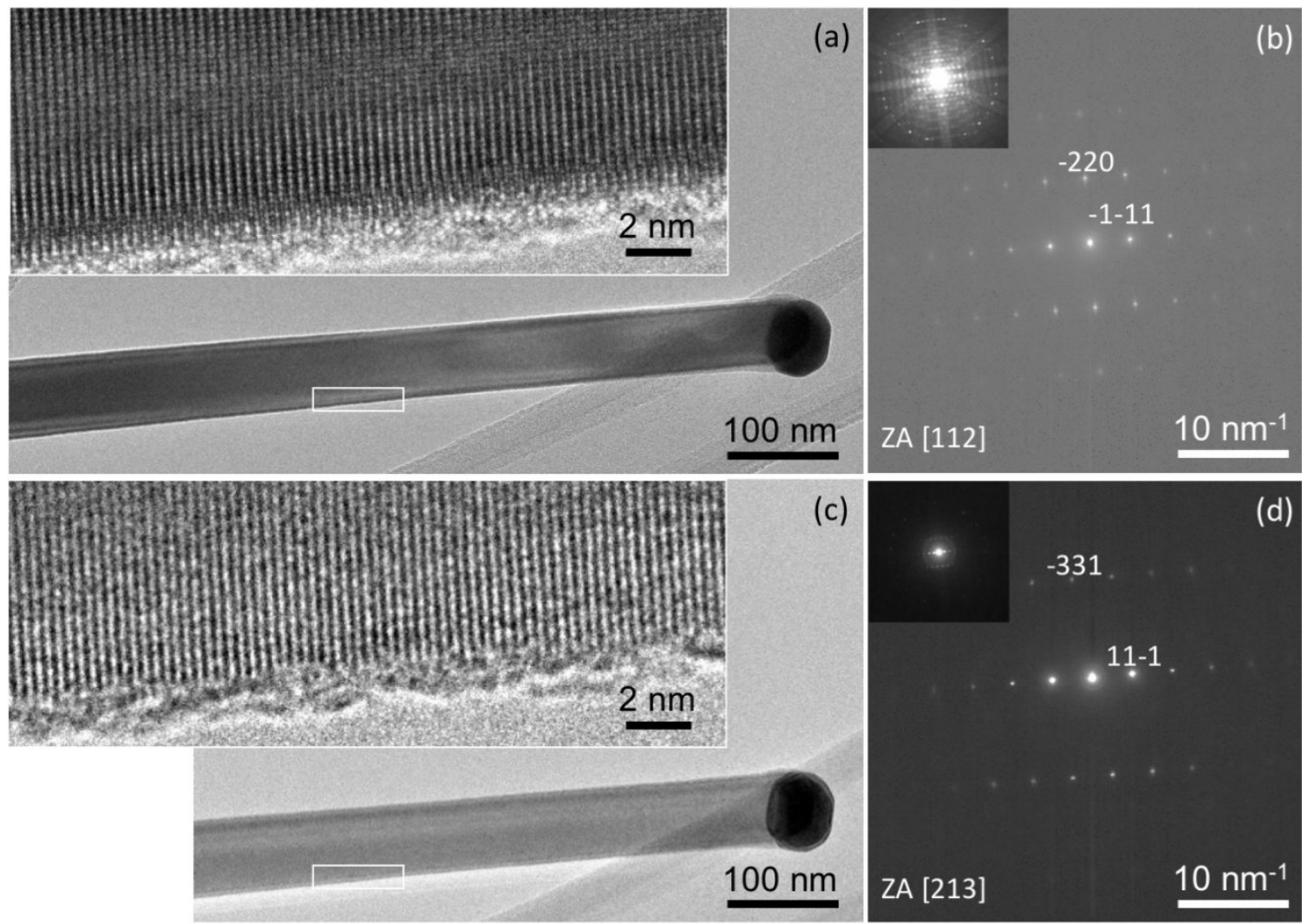

Figure S1. Morphology of a characteristic Ge nanowire. (a) TEM image of a typical Ge nanowire along the [112] zone axis. Inset: High-resolution TEM image of the nanowire part marked by the white rectangular frame in (a). (b) Selected area electron diffraction of the nanowire recorded along the [112] zone axis. Inset: Nanobeam electron diffraction pattern. (c) TEM image of the Ge nanowire after tilting by $11^{\circ}$ to the [213] zone axis. Inset: High-resolution TEM image of the nanowire part marked by the white rectangular frame in (c). (d) Selected area electron diffraction of the nanowire recorded along the [213] zone axis. Inset: Nanobeam electron diffraction pattern. Zone axes (ZA) are indicated in (b) and (d). 


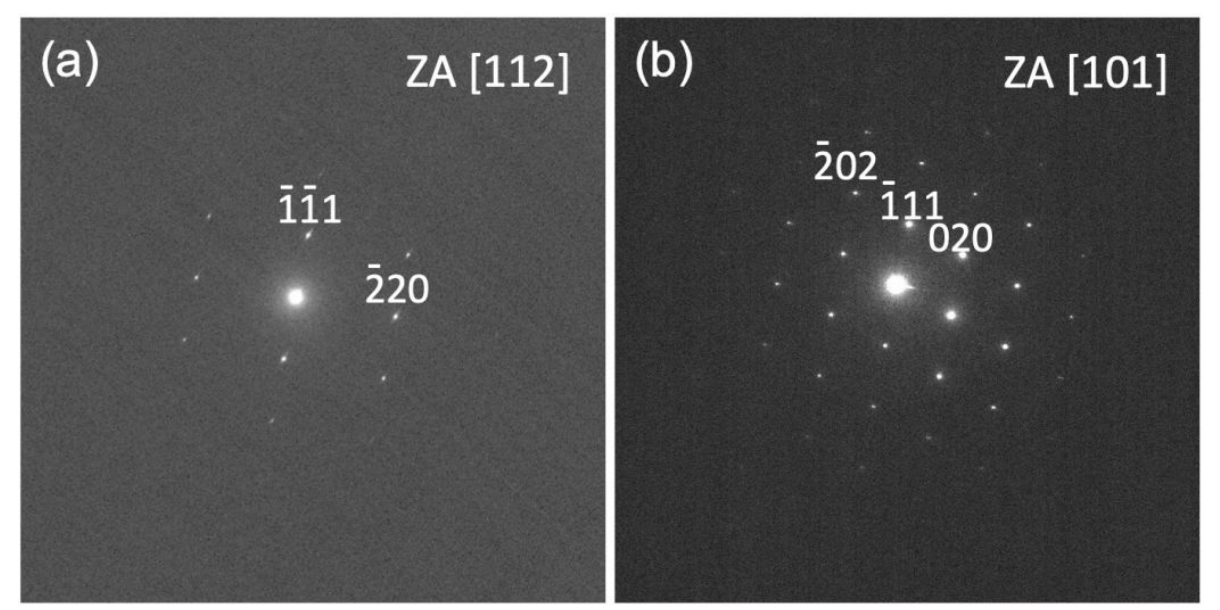

Figure S2. Electron diffraction on Ge nanowires along two different zone axes (ZA). (a) Diffraction pattern of a Ge nanowire along [112] zone axis. (b) Diffraction pattern of a Ge nanowire along [101] zone axis. Both patterns are indexed to singlecrystalline $\mathrm{Ge}$.
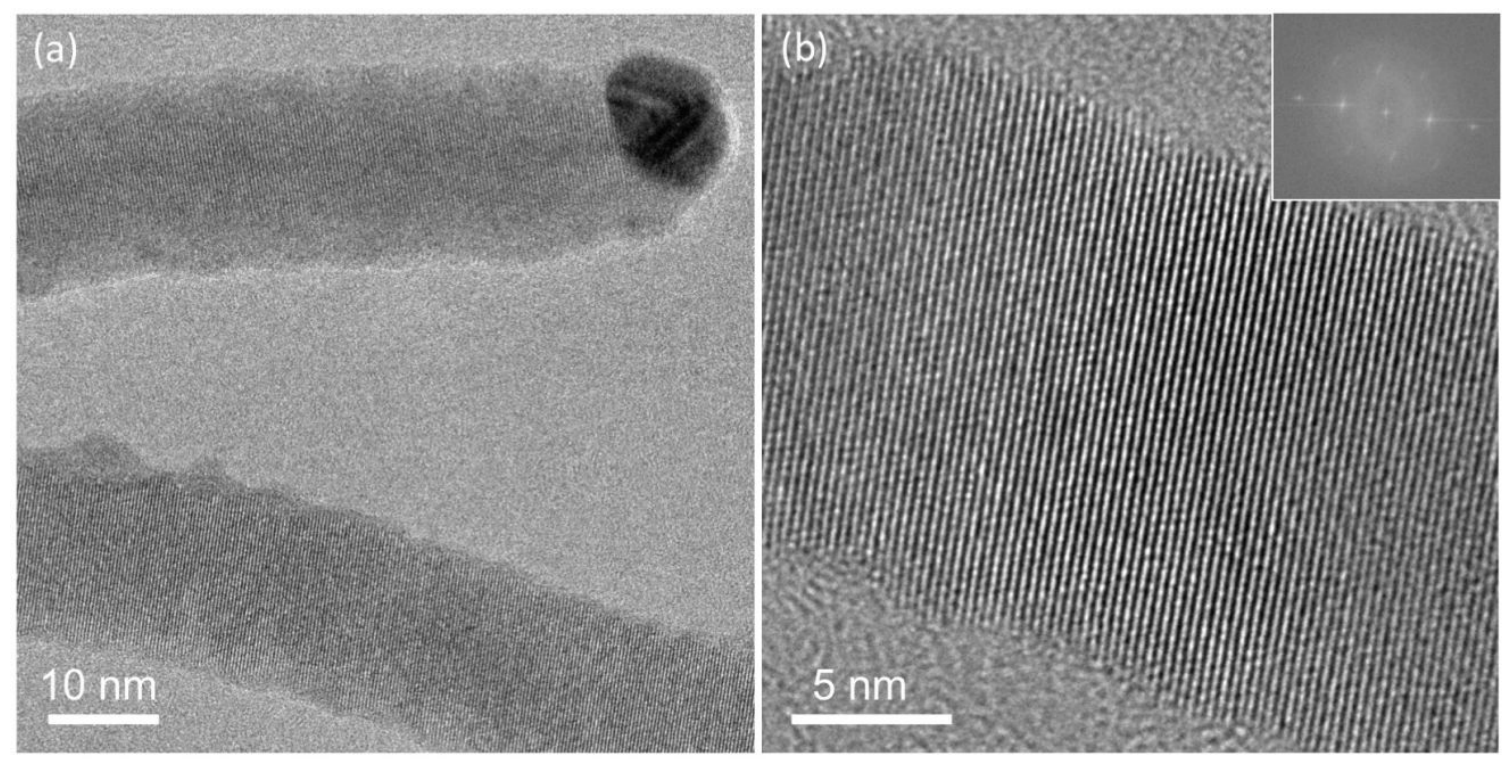

Figure S3. Crystal structure of Ge nanowires with diameters $<20 \mathrm{~nm}$. (a) Highresolution TEM image of two Ge nanowires ( $15 \mathrm{~nm}$ diameter). Both wires show $\{111\}$ planes perpendicular to the nanowire axis. (b) Higher magnification TEM image of part of the lower nanowire in (a) showing the [111] growth direction of the nanowire. Inset (top right): FFT of (b). 


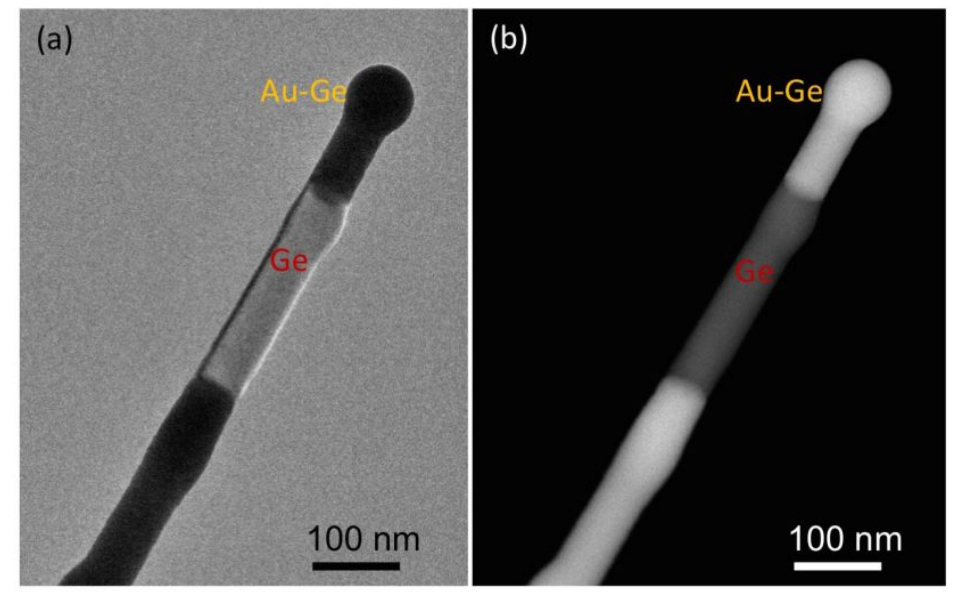

Figure S4. Ge-AuGe axial nanowire heterostructures. (a) TEM image of a segmented Ge-AuGe nanowire. (b) HAADF STEM (Z-contrast) image of the same nanowire.

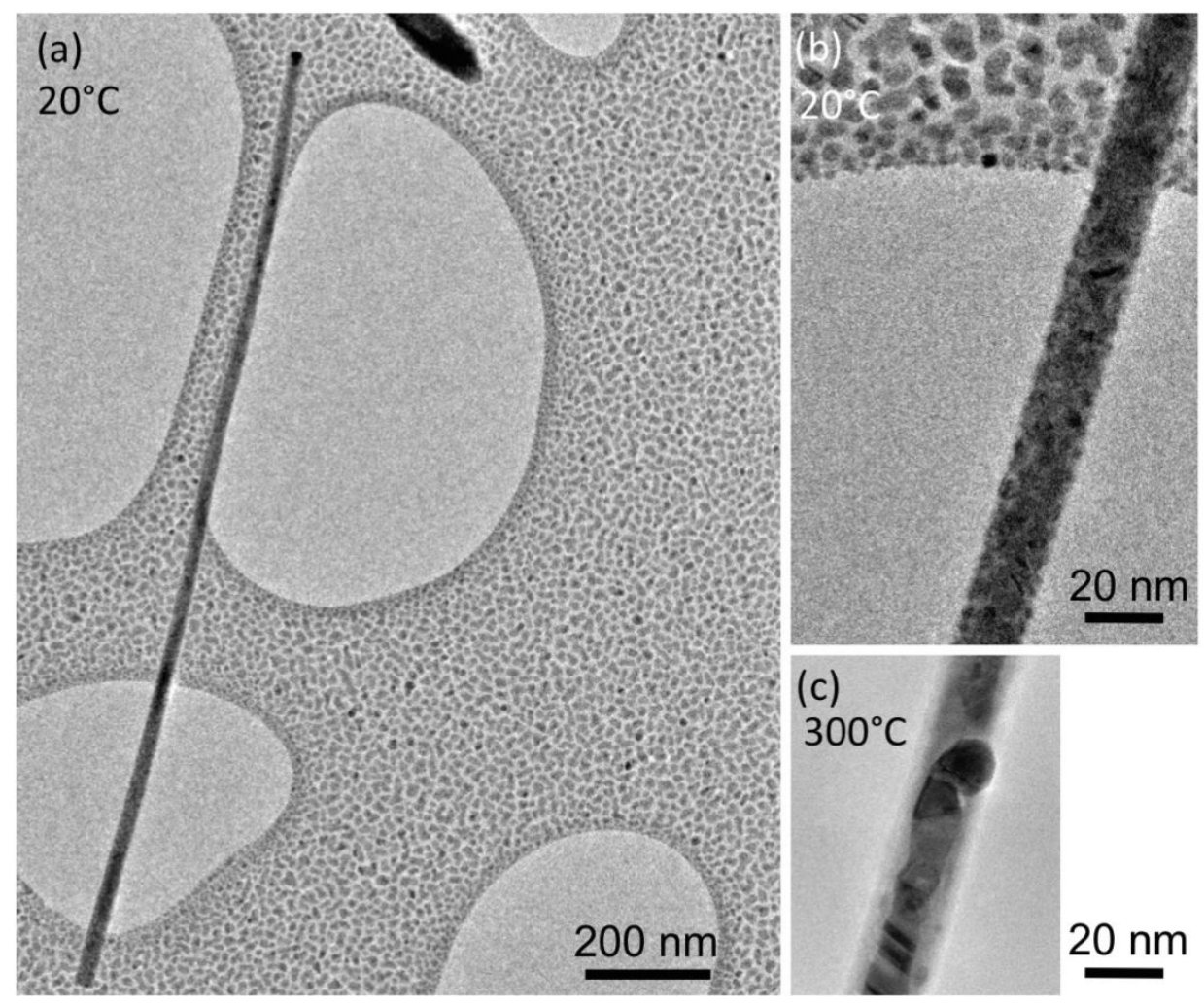

Figure S5. (a) Typical TEM image of a Ge nanowire on amorphous carbon grid after deposition of $\sim 1 \mathrm{~nm}$ thick carbon film and $5 \mathrm{~nm} \mathrm{Ag}$ at room temperature. The $\mathrm{Ag}$ film is polycrystalline and quasi-continuous. (b) Higher magnification TEM image of the nanowire showing the polycrystalline $\mathrm{Ag}$ film on its surface. (c) TEM image of a section of the same nanowire at $300^{\circ} \mathrm{C}$ showing the rearranging of the Ag on its surface. 

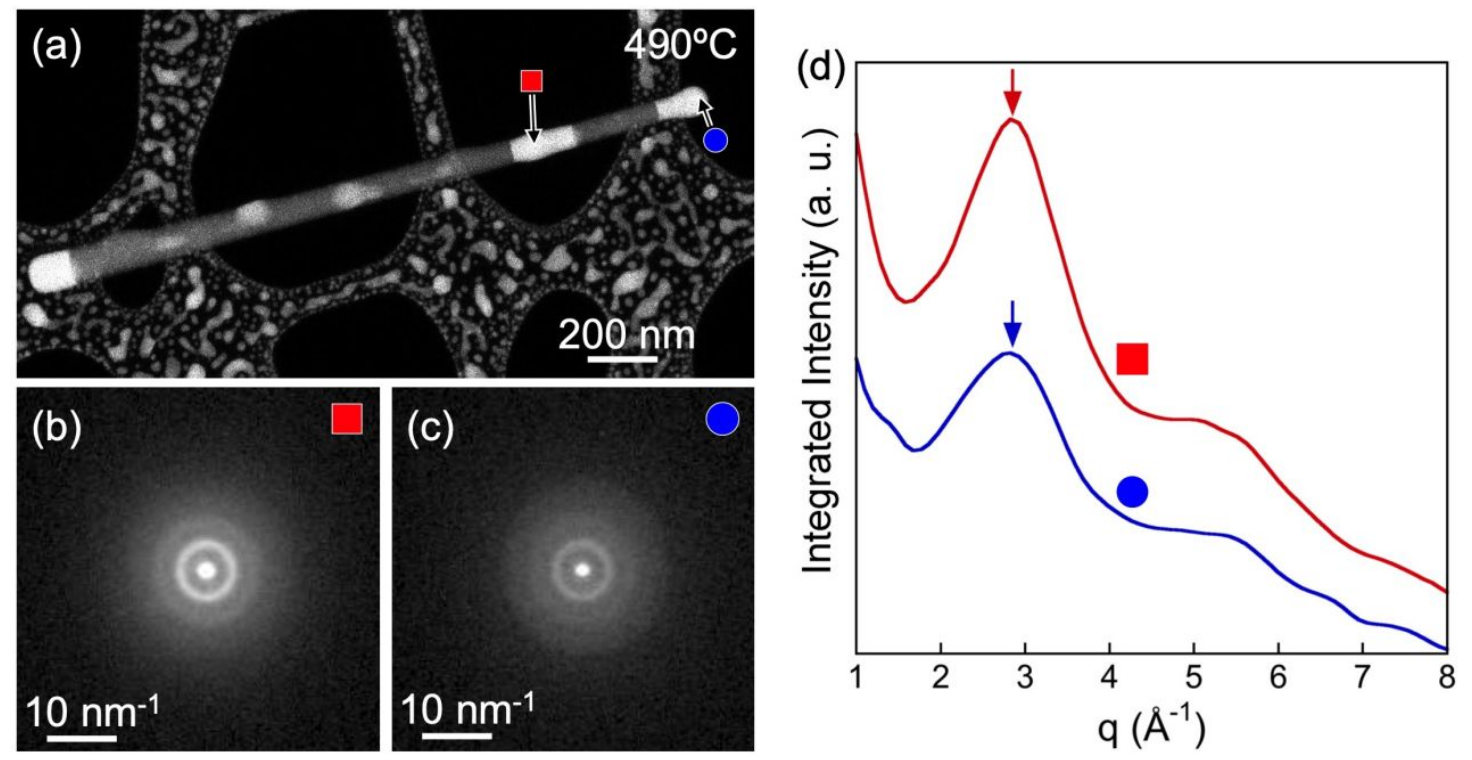

Figure S6. Comparison of nanobeam electron diffraction on AuGe melts at the nanowire tip and in alloy melt segments. (a) TEM image of an axially segmented nanowire $\left(65 \mathrm{~nm}\right.$ diameter) during annealing at $490^{\circ} \mathrm{C}$. Liquid AuGe melt segments are imaged with bright contrast, whereas Ge segments appear dark. (b) High-temperature electron diffraction pattern $\left(T=490^{\circ} \mathrm{C}\right)$ from the AuGe melt segment, obtained at the position marked by a red square in (a). (c) High-temperature electron diffraction pattern $\left(T=490^{\circ} \mathrm{C}\right.$ ) from the AuGe melt drop at the nanowire tip, obtained at the position marked by a blue circle in (a). (d) Radial distribution of the diffracted intensity of the liquid AuGe melts at the two positions along the nanowire (tip: blue circle; internal segment: red square) showing identical positions of the principal diffraction peak (arrows). 

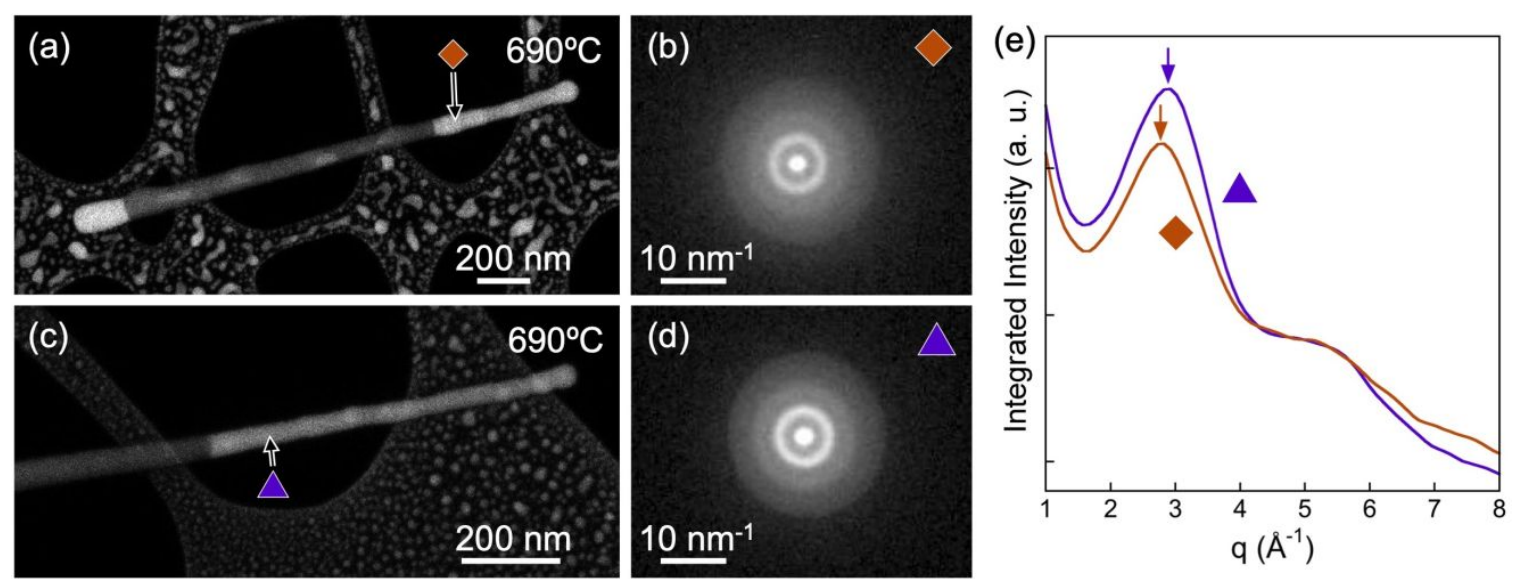

Figure S7. Comparison of nanobeam electron diffraction on AuGe melts in segments of thick and thin nanowires. (a) TEM image of a thick axially segmented nanowire $\left(65 \mathrm{~nm}\right.$ diameter) during annealing at $690^{\circ} \mathrm{C}$. Liquid AuGe melt segments are imaged with bright contrast, whereas Ge segments appear dark. (b) High-temperature electron diffraction pattern $\left(T=690^{\circ} \mathrm{C}\right)$ from the AuGe melt segment, obtained at the position marked by an orange diamond in (a). (c) TEM image of a thick axially segmented nanowire (30 nm diameter) during annealing at $690^{\circ} \mathrm{C}$. (d) High-temperature electron diffraction pattern $\left(T=690^{\circ} \mathrm{C}\right)$ from the AuGe melt segment, obtained at the position marked by a purple triangle in (c). (e) Radial distribution of the diffracted intensity of the liquid AuGe melts in thick (65 nm, diamond) and thin (30 nm, triangle) nanowires, showing the principal diffraction peak of the thinner wire shifted to larger spatial frequency (arrows), consistent with the results obtained on melt drops at the nanowire tips (Fig. 7 of the main text). 